\title{
Frontiers in the emerging development of blockchain and Bitcoin: visual research based on big data analysis
}

\author{
Chung-Lien Pan ${ }^{1}$, Zizhen Chen*1, Zhixiang-Zhou ${ }^{1}$, Zhuocheng Cai ${ }^{1}$, Xuanyan Liu ${ }^{1}$, Yiqi Lin ${ }^{1}$ \\ ${ }^{1}$ Nanfang College of Sun Yat-sen University,School of Accounting,Guangzhou, Guangdong
}

\begin{abstract}
In the era of the rapid development of information technology, the innovation of Fintech continues to send emerging research hotspots to the financial market. Based on the analysis of documents retrieved from the Web of Science database, this article provides a comprehensive data analysis and visualization of keywords such as "blockchain", "bitcoin", and "business and economic". Using big data analysis technology and visual presentation, the author analyzed the details of the author's keywords, popular organizations, countries, sources, and other key points of the correlation and external development status. Show the researchers the influence of the intersection of keywords, and point out the leading status of the organizations or countries with large resource occupancy in the research progress; at the same time, provide the researchers with an accurate grasp of the direction of the field and provide a reliable basis.
\end{abstract}

\section{Introduction}

Under the wave of financial technology, many new technologies and applications were born. Blockchain and Bitcoin are the new darlings of this era. Blockchain technology is the underlying technology of Bitcoin, and Bitcoin is the first amazing application of blockchain. People are scrambling to study the social and economic impact they bring. Through research, this article finds that blockchain technology has a broad impact on social development, and it has great value potential. Blockchain technology gives innovators the ability to create digital tokens to represent scarce assets, thus making it possible to reshape the pattern of entrepreneurship and innovation. Known as "one of the most revolutionary general technologies", it may have a profound impact on entrepreneurship and innovation [1]. Bitcoin-based distributed cryptocurrency technology and "smart contracts" that facilitate the digital implementation of its blockchain. It is called "the potential to replace the legal system to perform the core functions of the agreement [2]."

However, we also found many problems with them. The most obvious feature of Bitcoin at present is the large price fluctuation [3]. Short-term Bitcoin price behavior is prone to bubbles and crashes [4]. The fluctuation of the value of a virtual currency is likely to limit Its attractiveness to potential user groups [5]. The rise of blockchain technology may fundamentally disrupt the global economy [6], which is used for terrorist financing, theft, fraud, and ransomware [7]. The risks brought by the financial market activities carried out through the blockchain network are very similar to the risks in the current intermediary-based market: there are problems such as elasticity and financial stability, market distortions, and illegal activities [8]. Some people even label it "Untrusted Technology" [9].

Since then, we have asked, what should our government and related departments do in a decentralized application of new technology? Sauer suggested that "the most important task facing the central bank is to observe the growing influence of virtual currency and collect data on their acceptance [10]." This article is based on 70 articles collected by the Web of Science for scientific econometric analysis. Use VOSviewer to draw out relevant research information on this topic, and provide clear research directions for the government and relevant departments.

\section{Data and methods}

The bibliometric survey is different from the traditional literature review, it determines the most influential articles, journals, authors, and topics in the knowledge system. The results can provide a guide to the most frequently cited core articles and authors and how they link. To obtain relevant literature on blockchain and Bitcoin, this article first starts with the "Advanced Search" query of the (WoS) database and then explains the imaging protocol and strategy. The design is as follows:

- TS = ("Blockchain") and TS = ("Bitcoin") and SU = ("Business and Economics" or "Government and Law" or "Other Social Science Topics"). Various literacy terms were selected from the Oxford bibliography. As a result, 70 articles were retrieved in April 2020 and mapped using VOSviewer and the Python visualization package. 


\section{Visualization Research Andaper Analysis}

\subsection{Annual Trend}

As showed in figure 1, prior to 2017, the amount of research literature on blockchain and Bitcoin showed a slow rise. After 2017, the number of publications rose at a rapid rate and peaked in 2019. In 2019 alone, 33 papers were published. Figure 2 shows the number of annual citations. Since 2017, the number of citations has also increased dramatically, which should be related to the transaction price of Bitcoin hitting a record high at the end of 2017, which means the theme of blockchain and Bitcoin Received strong research motivation.



Fig1. Variation trend of citations

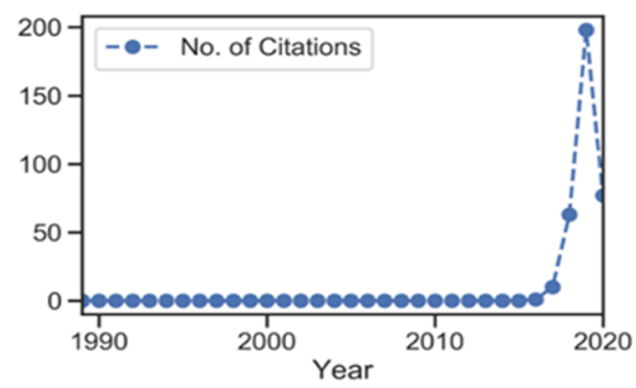

Fig2. Trends in number of publications

\subsection{WoS categories and research areas}

The classification of WoS is given in Table 1 (analysis of data interception accounting for more than 3\%). The top 5 are economics, business, business and finance, law, and management. The number of publications ranked in the top 5 accounts for a large proportion, accounting for more than $71 \%$ of the total number of publications. The lowerranking publications account for a low proportion and the number of articles is relatively scattered, indicating that there are obvious clusters in the fields of economics, business, finance, law, and management. Other categories include social sciences, interdisciplinary, computer science and information systems, etc. This emerging category of applications is also pouring into traditional research areas.

Typical research fields are shown in Table 2, and 6 research fields accounting for more than $4 \%$ are selected for analysis. The top 3 fields account for 77.7 percent of all published papers, indicating a higher concentration of business and economics, government and law, social sciences - other topics. The top 6 places in the field are roughly the same as in the WoS category, but the concentration is higher than the WoS category. Besides, compared with the WoS category, more diverse research fields have emerged, such as government, public administration, etc.

Table1. Top WoS Categories

\begin{tabular}{|c|c|c|}
\hline WoS categories & $\begin{array}{c}\text { Number of } \\
\text { articles }\end{array}$ & 109 in total \\
\hline Economics & 22 & $20.18 \%$ \\
\hline Business & 19 & $17.43 \%$ \\
\hline Business, Finance & 14 & $12.84 \%$ \\
\hline Law & 12 & $11.01 \%$ \\
\hline Management & 11 & $10.09 \%$ \\
\hline Social Sciences, Interdisciplinary & 4 & $3.67 \%$ \\
\hline Regional \& Urban Planning & 4 & $3.67 \%$ \\
\hline Sociology & 4 & $3.67 \%$ \\
\hline $\begin{array}{c}\text { Computer Science, Information } \\
\text { Systems }\end{array}$ & 4 & $3.67 \%$ \\
\hline \multicolumn{3}{|c|}{ Table2. Top Research Areas } \\
\hline Research areas & $\begin{array}{c}\text { Number of } \\
\text { articles }\end{array}$ & $\%$ \\
\hline Business \& Economics & 53 & $56.38 \%$ \\
\hline Government \& Law & 13 & $13.83 \%$ \\
\hline Social Sciences - Other Topics & 7 & $7.45 \%$ \\
\hline Computer Science & 4 & $4.26 \%$ \\
\hline Public Administration & 4 & $4.26 \%$ \\
\hline Sociology & 4 & $4.26 \%$ \\
\hline Others & 9 & $9.57 \%$ \\
\hline
\end{tabular}

\subsection{Top organizations}

In this study, the author's mechanism distribution was tested to determine its geographical distribution. Table 3 lists all organizations accounting for more than $3 \%$. The top 4 are located in the USA, England, Australia, and Canada, accounting for 56.1 percent of the total. It can be observed that most of these organizations that occupy a large proportion are located in places with developed economies and sound systems. These developed countries and regions have a high level of education, pay attention to the power of science and technology, and improve relevant laws. These conditions have created a good environment for the rapid development of blockchain.

Table3. Top Countries

\begin{tabular}{ccc}
\hline Country & $\begin{array}{c}\text { Number of } \\
\text { articles }\end{array}$ & $\%$ \\
\hline USA & 25 & $25.51 \%$ \\
England & 14 & $14.29 \%$ \\
Australia & 9 & $9.18 \%$ \\
Canada & 7 & $7.14 \%$ \\
Germany & 3 & $3.06 \%$ \\
Italy & 3 & $3.06 \%$ \\
Netherlands & 3 & $3.06 \%$ \\
China & 3 & $3.06 \%$ \\
Spain & 3 & $3.06 \%$ \\
Switzerland & 3 & $3.06 \%$ \\
\hline
\end{tabular}




\begin{tabular}{rrr}
\hline Others & 25 & $25.51 \%$ \\
\hline
\end{tabular}

\subsection{Popular Publications}

In the article with the highest citation rate, Kiviat, Trevor I pointed out that blockchain technology is adaptable, and policymakers should encourage regulation aimed at reducing the risk of this powerful technology, and should carefully and precisely adjust the scope of regulation [11]. Scholars such as Werbach believe that when smart contracts will eventually be widely adopted, the only way to prevent smart contracts from having serious negative consequences may be government regulation [12].

The other top 10 most cited articles also focus on the impact of Bitcoin or blockchain technology on business and finance, law and management, etc [13][14][15][16]. The visualization results did not find citation rankings for top scholars, nor did it find significant author groups, which meant that there was no distributed citation network for top scholars, and collaboration links were very sparse.

\section{5 cluster}

In this paper, VOSviewer software is used to build a cocitation map of author keywords, as shown in Figure 3, to study the relationship between related author keywords and this topic. The feature of this software is that it can display the evolution of a certain field in a knowledge map, and provide intuitive analysis assistance for researchers to identify the research hotspot and frontier direction in a certain field. After statistics, there are 212 author keywords in total. In this paper, the first 29 important keywords are selected for visual analysis, and they are split into 7 color groups in Figure 3.

The Green ethnic group is the most obvious ethnic group in the picture. There are two levels of differentiation in the nodes within the group: "blockchain" and "bitcoin" have become the most popular cited keywords of the group, which is enough to show that researchers attach great importance to this field under the promotion of financial science and technology. The red community involves such topics as "smart contract", "trust", "relationship contract", etc. In the purple community, including the "privacy", "data protection" and other key nodes. Purple ethnic groups act as a bridge between green and red ethnic groups, and they are closely linked. The node representing "digital cryptocurrency" in the Yellow ethnic group is the most important in the ethnic group, and also the most closely connected communication node with the outside world, which provides a way for the nodes representing "governance" and "digital assets" in the ethnic group to connect with the outside world. The blue group includes "ICO company", "token trading", "financial technology" and other topics. Although the number of citations is not significant, they are still actively linked and cited externally. Finally, we focus on the two groups with only one key node at the bottom and right of the visualization diagram. They are the lake blue group containing the "blockchain technology" node and the orange group containing the "activity research" node. Although they do not have many advantages in terms of citation and external correlation density, they can independently form an important keyword to appear in the visualization chart, which shows that the key research point of emerging influx is the details that can not be ignored in the future in this field.



Fig3. Co-citation of author's key words

As shown in Figure 4, the relationship described in this field involves 9 clusters, covering 19 countries. The node representing "America" in the blue group is the largest in the figure. The red ethnic groups headed by "Australia" include Malaysia, Switzerland, and Denmark. This data branch not only has a close relationship with the data of the United States, but also connects the data systems of Australia and Switzerland, and also enables the Yellow ethnic groups to play a role in connecting the red and blue ethnic groups. Including Spain, Italy, Indonesia, and other branches of the green ethnic group not only have a significant connection with the blue ethnic group but also connect with the red ethnic group. The orange ethnic group representing Norway, the lake blue ethnic group representing Belgium, and the brown ethnic group representing South Korea are the other related data objects shown in the figure. Although the data proportion is very small, the external related communication is realized by connecting with the main nodes of other ethnic groups. The purple group contains the UK and Austria, and the UK is the second-largest node in the figure.

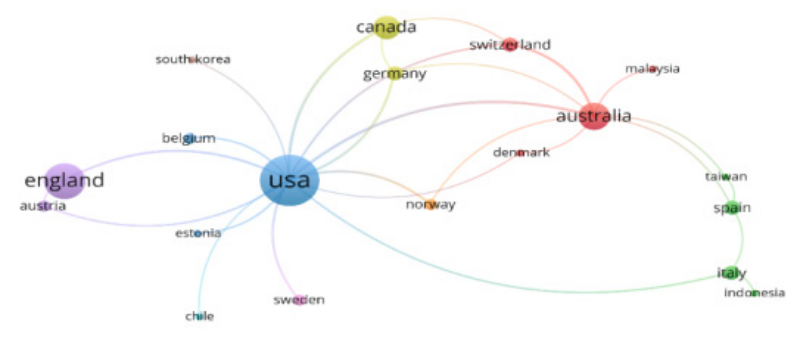

Fig4. National citation relationship

The organization co-citation relationship is shown in Figure 5, which shows the co-citation relationship of popular organizations. The whole picture is composed of five color groups, among which the popular organizations with large node share are mainly concentrated in four groups, namely Queen Mary College of London University, University of Genoa, Federal Reserve Bank of St. Louis, and Stevens Institute of technology. According to the color classification of ethnic groups, red ethnic groups account for the largest proportion, with close and orderly internal relations, and they are the only active ethnic groups in the legend that can have co-citation 
relationships with the three ethnic groups. Secondly, the proportion of the green population in the number of nodes is also relatively dominant and plays the role of a bridge between the red and blue populations. Yellow and purple ethnic groups are located in the branch of the red ethnic group, which is worthy of attention.

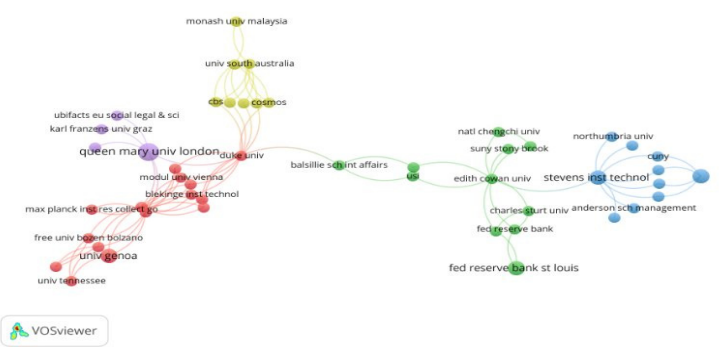

Fig5. organization co citation diagram

Figure 6 shows 19 publishing channels, among which "European financial journal", "business ethics journal", "management information journal" and "Duke Law Journal" obviously occupy the most important position, and they are actively related to the outside world. Compared with other ethnic groups, the proportion of red ethnic groups is higher, and each node in the ethnic group is in a leading position in terms of quantity and citation relationship. For the blue and green ethnic groups in the red ethnic group, although there are a few representative important nodes within the ethnic group, they do not show uniform co-citation intensity as a whole. The purple ethnic group in the middle is the middle hub connecting the green ethnic group and the red ethnic group. Although it has no advantage in proportion, it is a key link that cannot be ignored.

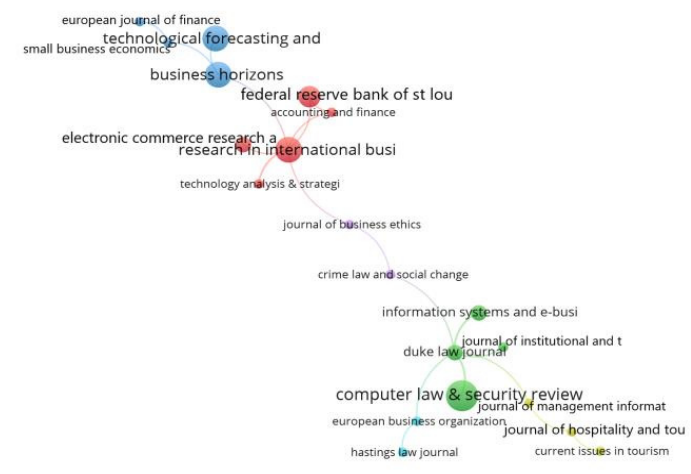

Fig6. publication co citation diagram

\section{Conclusion}

The financial market is changing rapidly, and blockchain and bitcoin, as emerging research fields, are rushing into people's research perspective with a rapid development trend. After analysis and research in this article, it is found that the field is still in a state of new entrants. Related research is gaining momentum, but it has not yet reached the stage of the qualitative outbreak, which is a key point for emerging researchers that deserves high attention. And the study found that due to its inherent resource advantages, traditional journals or organizations in Europe and America still have a high degree of acumen in the emerging research field. Therefore, for other researchers, even if the opportunities for blockchain and Bitcoin research are challenges, it is necessary to actively establish a variety of external communication channels while constantly discovering new problems. The combination of current cutting-edge analytical technology and multidisciplinary research methods will inevitably inject more powerful momentum into this field of research.

\section{Acknowledgment}

We thank our colleague Han-Teng Liao and Zhichao Xu who provided expertise and insight into the bibliometric data that greatly helped the research.

\section{References}

1. Chen, Y (2018) Blockchain tokens and the potential democratization of entrepreneurship and innovation

2. Werbach, K; Cornell, N (2017) CONTRACTS EX MACHINA

3. Lee, K; Ulkuatam, S; Beling, P; Scherer, W (2018) Generating Synthetic Bitcoin Transactions and Predicting Market Price Movement via Inverse Reinforcement Learning and Agent-Based Modeling

4. Akcora, CG; Dixon, MF; Gel, YR; Kantarcioglu, M ( 2018 ) Bitcoin risk modeling with blockchain graphs

5. Zharova, A; Lloyd, I (2018) An examination of the experience of cryptocurrency use in Russia. In search of better practice

6. Kwok, AOJ; Koh, SGM (2019) The Pricing and Performance of Cryptocurrency

7. Schmitz, J; Leoni, G (2019) Accounting and Auditing at the Time of Blockchain Technology: A

8. Paech, P (2017) The Governance of Blockchain Financial Networks

9. Kiviat, TI (2015) BEYOND BITCOIN: ISSUES IN REGULATING BLOCKCHAIN TRANSACTIONS

10. Abraham, J; Sutiksno, DU; Kurniasih, N; Warokka, A (2018) Acceptance and Penetration of Bitcoin: The Role of Psychological Distance and National Culture

11. Trevor I. Kiviat, Beyond Bitcoin: Issues in Regulating Blockchain Transactions, 65 Duke Law Journal 569608 (2015) Available at:https://scholarship.law.duke.edu/dlj/vol65/iss3/4

12. Werbach, K., \& Cornell, N. (2017). Contracts Ex Machina. Duke Law Journal, 67(2), 313-382.

13. Davidson, Sinclair; De Filippi, Primavera; Potts, Jason(2018) Blockchains and the economic institutions of capitalism

14. Dierksmeier, Claus; Seele, Peter(2018) Cryptocurrencies and Business Ethics

15. Paech, Philipp(2017) The Governance of Blockchain Financial Networks 
16. Cai, Cynthia Weiyi(2018) Disruption of financial intermediation by FinTech: a review on crowdfunding and blockchain. 